\title{
Failover for Mobile Routers: A Vision of Resilient Ambience
}

\author{
Eranga Perera ${ }^{1}$, Aruna Seneviratne ${ }^{2}$, Roksana Boreli², Michael Eyrich ${ }^{3}$, Michael Wolf ${ }^{4}$ Tim Leinmüller $^{4}$ \\ ${ }^{1}$ Dept. of Electrical Engineering, University of New South Wales, \\ Sydney, Australia \\ eranga@mobqos.ee.unsw.edu.au \\ ${ }^{2}$ National Information and Communication Technologies Australia (NICTA) \\ Bay 15, Locomotive Workshop, Australian Technology Park, Eveleigh, \\ NSW 1430, Australia \\ \{aruna, Roksana.Boreli\}@nicta.com.au \\ ${ }^{3}$ Technical University of Berlin, TKN, \\ Germany \\ eyrich@tkn.tu-berlin.de \\ ${ }^{4}$ DaimlerChrysler AG, Researchand Technology / Vehicle IT and services, \\ P.O. Box 2360, \\ Ulm, Germany \\ \{michael.m.wolf, tim.leinmueller\}@daimlerchrysler.com
}

\begin{abstract}
The ambient networking approach includes the flexibility of every end system to be not just a node but also an entire network. The end user entities are, in the majority of use cases, mobile and they operate in a highly dynamic environment. The exponential growth of wireless devices and services contributes to the increasing number of these dynamic mobile networks, whose changeable characteristics in turn contribute to the high probability of failures. To maintain the high level of connectivity required for the overall ambient networks environment, it is imperative to maintain the same level of connectivity in various network parts. In this paper we consider the Mobile Router related failures that could occur in today's mobile networks and describe how the ambient networking environment with its in-built enhanced failover management functionality has a potential to create resilient networks.
\end{abstract}

\section{Introduction}

AMBIENT networking is geared towards increasing competition and cooperation in an environment populated by a multitude of user devices, wireless technologies, network operators and business entities. This architecture aims to extend all IP networks with three fundamental requirements of today's networking world. These requirements include dynamic network composition, mobility and heterogeneity. By encompassing these notions the Ambient Networks (AN) project [1] strives to achieve horizontally structured mobile systems that offer common control functions to a wide range of different applications and air interface technologies. In such an environment the user expectation for high availability is inevitable and providing this is vital in order to be viable in today's market. We therefore consider failover management to be an essential requirement for resilient ambient networking.

In this paper, we first describe the need for failover management of Mobile Routers. Section 3 will carry an introduction to the AN architecture from the perspective of failover management. Section 4 will investigate different types of mobile networking scenarios in order to present the problem. The requirements elicited from the scenarios will be stated in Section 5. In section 6 we will delve deeper into the Ambient Networks concepts to illustrate how this architecture can fulfill the elicited requirements. This will be followed by related work. Finally we will conclude the paper. 


\section{The Motivations}

\subsection{Why failover management as opposed to simple fault tolerance?}

It is important to recognize that any system is prone to failures and this is especially applicable to wireless systems since wireless channels have adverse characteristics such as limited bandwidth and high jitter. Another contributing factor for unanticipated failures in a mobile networking environment is the dynamicity. At any given time a failure could occur due to the sheer number of nodes attempting to connect to the Internet via a Mobile Router. Therefore in order to face such challenges transparent to the users there needs to be high-quality failure recovery mechanisms.

\subsection{Why investigating Mobile Routers as opposed to any other entity in the AN architecture?}

AN can be defined as an All-IP based mobile network that adopts the much needed technological innovations of the beyond 3G networks. This relation of ANs to mobile networks creates the need for us to consider the existing IP based mobility architectures. One such architecture is the NEtwork MObility (NEMO) [2] architecture. In the AN framework, Mobile Routers facilitate the mobility of the entire networks and provide a way to mount a complete network into the Internet's addressing hierarchy just as a mobile node can be mounted using Mobile IP [3]. Even though Mobile Routers bring about many benefits for the entire mobility environment, they create a single point of failure for an entire network in contrast to the failure of a single node. This motivated us to look into Mobile Routers when considering a resilient AN mobility management architecture.

\section{Ambient Networking Approach}

One of the main challenges in today's networking world is how to address mobility, heterogeneity and the integration of networks without having to plug and play various services in an ad-hoc manner. In order to answer this question the AN architecture introduces a novel concept of an Ambient Control Space (ACS). The Ambient Control Space is a vision of an overlay network that would bring about the control functions for heterogeneous networks under one umbrella. By introducing a 'thick' control plane in Ambient Networks as opposed to the 'thin' control plane of the IP networks, the ACS would pave the way for seamless mobility. By making use of this well defined Ambient Control Space (ACS) via a well defined external interface named as the Ambient Network Interface (ANI) users can expose their communication resources to other users. The most primitive building block of an $\mathrm{AN}$ is a physical cluster, which can be defined as a group of nodes (or just one node) that are physically close to each other, are likely to stay near each other and are able to communicate. If the nodes of such a physical cluster are aware of each other then it can be considered as a routing group. This "awareness" involves, besides others, functionalities like address and mobility management, AAA, service and gateway discovery, and failover functionality. Addressing the failover during the architectural design phase allows incorporating its requirements in the solution design rather than patching the system by adding e.g. hot-standby proxy-units. Failover management will utilize intra- and inter-ACS communication depending on whether the involved entities are part of the same or different AN domain. In the following figure we have depicted some of the core functional areas being researched in the AN project. The 'Failover for Mobile Routers' is a sub function of 'Mobility' functions. 


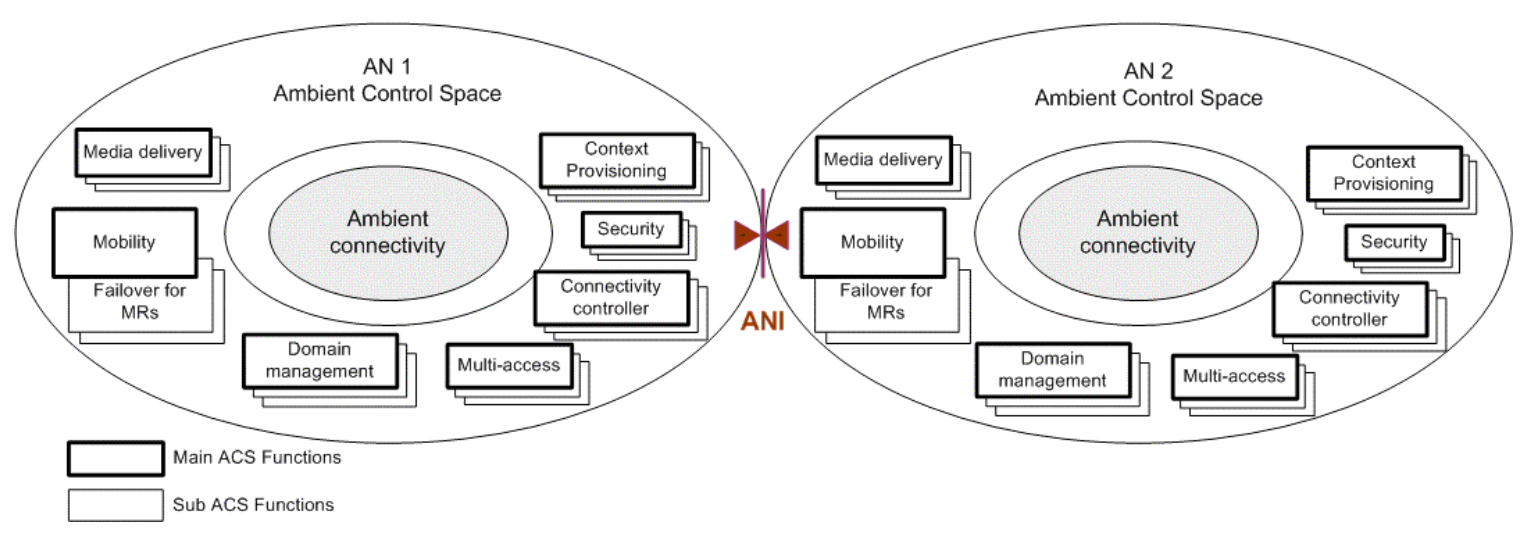

Fig. 1. Ambient Control Space

The functional area "Mobility" itself is organized in a toolbox like manner that allows multiple mechanisms to co-exist. It distinguishes between basic mobility management and advanced mobility management functions. The basic mobility management contains among others trigger processing, location management, session continuity and handover mechanisms. The latter type of mobility management in contrast provides add-on functionalities like multi-homing, context dependant handovers and inter-domain seamless handovers making use of inputs and outputs from other functional areas. Naturally the failover management belongs to the advanced mobility management functions.

In the next section we carry a problem description by making use of three different scenarios. This is followed by an analysis of how these problems can be tackled using the functionalities offered by the ACS.

\section{Scenarios and Problem Description}

We introduce three types of mobile networks in considering failover management, namely managed routing groups, unmanaged routing groups and hybrid routing groups. The former type of mobile network has a specific node as Mobile Router. The second type of mobile network has no such specific node and any device belonging to the network plays the role of a Mobile Router on the fly and the hybrid type has both these types of nodes which come into play in failover management.

Examples of managed routing grouped networks are public transportation systems with on-board Mobile Routers for Internet connectivity. The routers deployed on such networks are commercially available routers. We identify these routers as Specific Mobile Routers (SMR).

The unmanaged routing groups bring about the notion of any capable device taking on the task of providing Internet connectivity for the rest of the nodes in the group. Personal Area Networks (PAN) fall within this category of routing groups. In an unmanaged routing group the devices that play the role of a router dynamically are identified as UnSpecific Mobile Routers (USMR) within the context of this paper.

Private cars which have a combination of designated MRs (SMR) and external devices (such as passengers or owner's mobile devices) that could play the role of a MR on the fly (USMR) would constitute a hybrid type of routing group.

We use 3 scenarios in order to describe the issues associated with each kind routing group with SMRs and USMRs. 


\subsection{Scenario 1: Highlighting failures of SMRs}
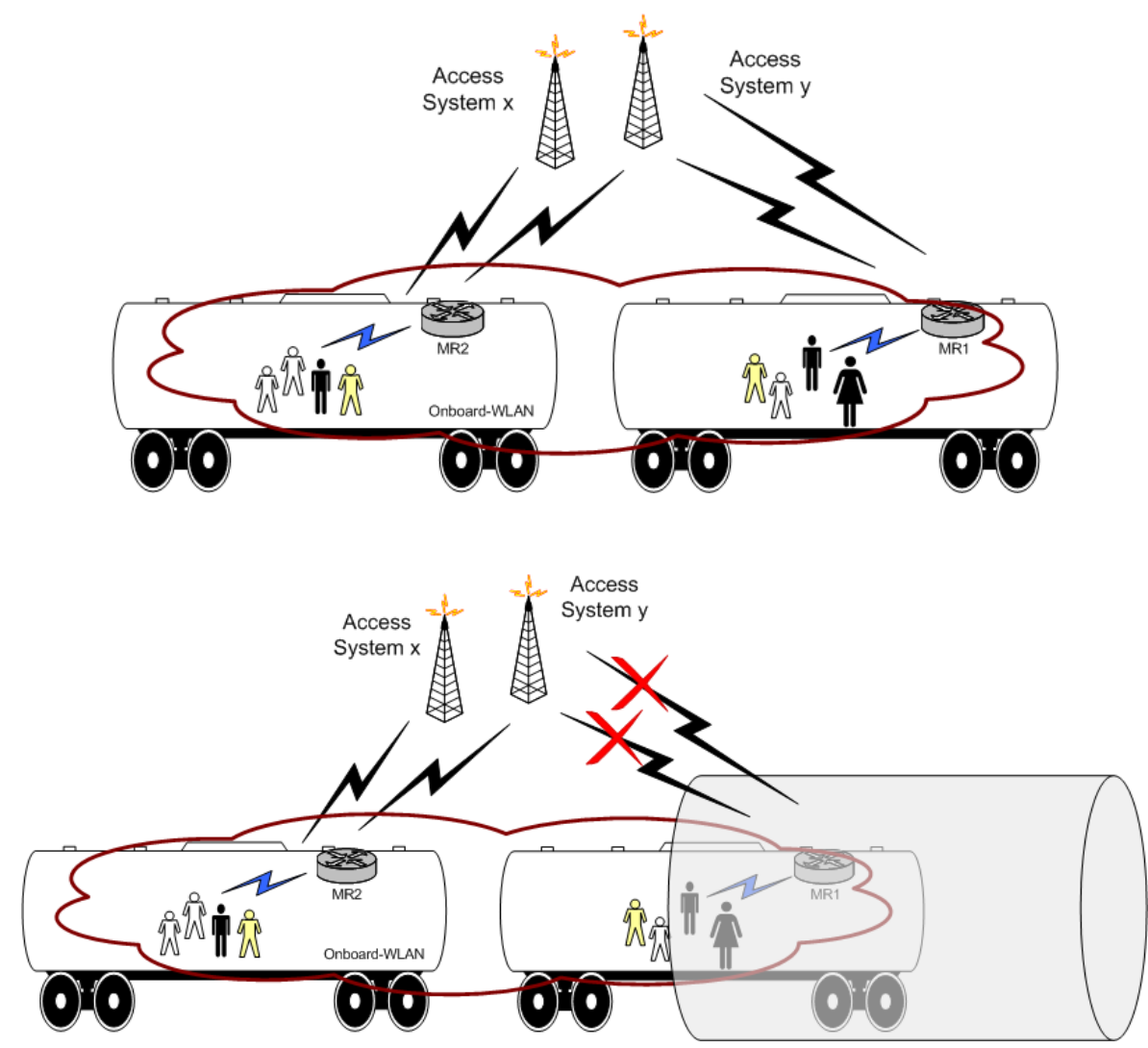

Fig. 2. Managed Routing Group with SMRs

Upon entering the tunnel the SMR1 experiences an outage which causes a connection failure with the base station. In such a case the SMR2 will provide failover for SMR1. It is important to note that these outages to the base stations could be quite common not only due to physical tunnels but also due to congestion, unavailability of channels etc. Another angle to this scenario is where these routers are connected to different providers for more robustness. Suppose in that case if one of the routers has a potential outage with its provider then the other router with the different provider would need to take over.

\subsection{Scenario 2: Highlighting failures of USMRs}
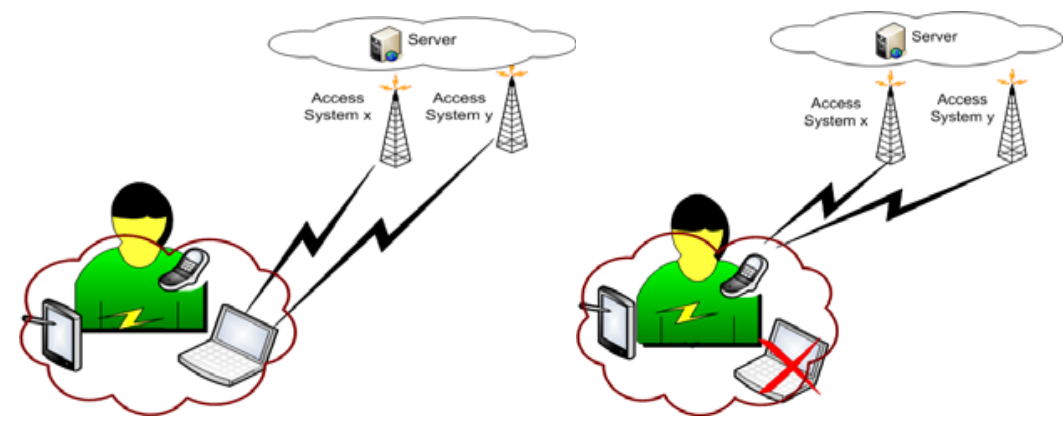

Fig. 3. Unmanaged routing group with USMRs

This scenario depicts an unmanaged routing group (PAN) with many devices such as a PDA, mobile phone, laptop a digital camera and a MP3 player. Suppose the user is downloading an MP3 file to her MP3 
player via the laptop. (In this case the laptop is playing the role of the MR). If the laptop fails for some reason for example by draining its battery then some other device of the PAN needs to take over routing of the mobile network. This scenario depicts the case of a USMR providing failover for another USMR.

Furthermore consider the case where the home network of the mobile phone belongs to the user's office network. In this case the failover mechanisms would become more complex.

\subsection{Scenario3: Failures in a hybrid combination of SMRs and USMRs}
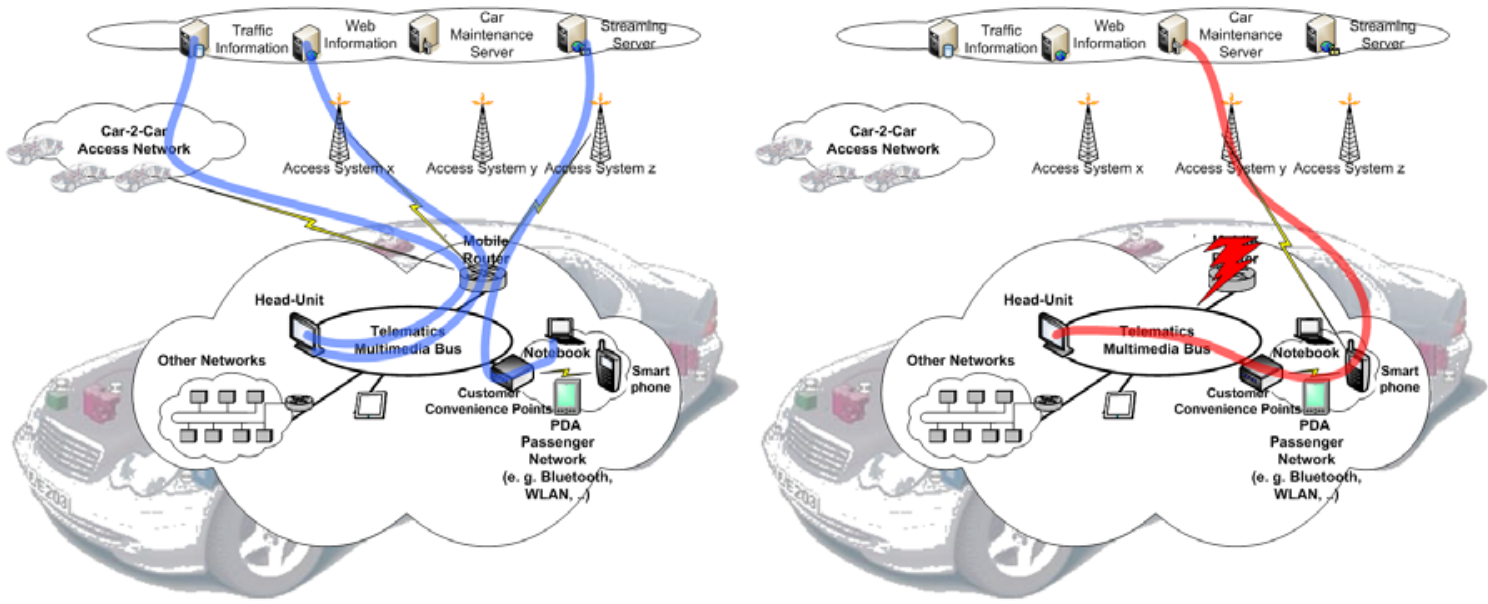

Fig. 4. Hybrid routing group with SMRs and USMRs

In this scenario we have depicted the case where there is a single SMR deployed in the car (mainly due cost reasons) and the failure of the SMR requiring another device in the car, an USMR to come into play. Moreover this scenario illustrates the case of the USMR providing failover for only a single connection out of the connections maintained by the SMR.

\section{Requirements Elicitation}

In this section we identify the requirements pertaining to each of the scenarios described in section 3 . Even though most of these requirements are applicable to all of the failover scenarios, this method of requirement elicitation demonstrates that the failover mechanisms need to be adaptive to a diverse range of mobility environments with a variety of devices, resources, environmental conditions etc.

\subsection{Scenario 1}

Mobility Predictions A replacement Mobile Router should be activated best shortly before the current Mobile Router is deactivated for whatever reason in order to achieve seamless failovers. Route predictability is an exploitable characteristic of public transportation systems that we can use to avoid disruptions caused by unexpected outages. It is possible to gather information pertaining to link outages caused by interference, time of the day, hot spot areas, weather etc. This information together with location predictions of vehicles can be used to minimize the 'mean time to detect' contributing to seamless failover mechanisms.

Bandwidth consumption in failure detection Two main scarce resources in mobility management are low data rates and power. It is fair to assume that SMRs would have the necessary power (that is power supplied by the vehicle) in order to perform its routing functionalities. Therefore in the public transportation mobility environments the scarce resource pertaining to SMRs is the consumption of bandwidth. 
Cost of providing unperturbed Internet connectivity In this scenario by deploying Mobile Routers redundantly would increase the probability of providing unperturbed Internet connectivity. But this would not be ideal because it would mean that this cost would contribute to increasing the charging for onboard connectivity from passengers. Therefore it is necessary to find the minimum number of Mobile Routers needed in order to achieve an optimal recovery from a failure. Furthermore consider the case of a 'bus'. Since it is possible to cover an area of a bus with one MR deploying another MR as a backup is not cost effective. In such cases it is necessary to utilize cooperating Mobile Routers in the vicinity for failover mechanisms. These cooperating MRs might be from a different administrative, naming, security, addressing or mobility domain.

\subsection{Scenario 2}

Power consumption in failure detection The typical nodes of a moving PAN would be laptops, PDAs, mobile phones etc. All these devices would typically rely on battery power. Therefore power consumption becomes a more imperative issue with USMRs than with SMRs that would be powered by the vehicle. For example if in order to minimize the 'mean time to detect a failure', if the devices continuously send liveliness messages this would not be worthwhile in terms of the power consumption.

Discovering candidate backup Mobile Routers In an USMR environment there are no nodes specifically assigned to be MRs, that is potential Mobile Routers are not known by definition. Therefore a discovery mechanism for devices that can take on the mobility management in case the primary device fails should be in situ.

State synchronization When the new device takes over it has to be synchronized with the device that was playing the role of the Mobile Router. In the USMRs case if the other potential back up devices are to be synchronized with the MR constantly this would dissipate power unnecessarily. On the other hand if state synchronization is not done in a timely manner this would cause delays and the failover will not be seamless.

\subsection{Scenario 3}

Connection handling and prioritization The USMR in this case the PDA is not as capable as the SMR deployed in the automobile. Consequently it can support only a few connections out of the connections handled by the SMR. In such a case there needs to be priority based QoS interactions in the failover management procedures. Furthermore the QoS needs to be dynamically adaptive. Suppose at any given time the PDA is able to handle another connection and is prepared to do so, then the QoS mechanisms should adapt to the availability.

\section{Failover Management as a Functionality of the Mobility Functional Area within the Ambient Control Space}

The following section explains how the failover management becomes an easily achieved functionality within the ACS mobility Functionality Area (FA). The proposed AN architecture does not suggest any mechanisms to replace the networks that are in existence today, but rather a mechanism to coalesce today's networks in a coherent manner. By relating the elicited requirements to a failover functional model we demonstrate how the AN architecture facilitates the fulfillment of a seamless failover for Mobile Routers. 


\subsection{Failover Functional Model}

The failover model (fig. 6) shows the functions and the functional flow that need to be in place for a complete seamless failover. It relies on the input from other mobility functions and also from the other functions supported by the generic Ambient Control Space functional areas. The aim is to react as early as possible to failover events and to start the appropriate actions. It is evident that the current IP architecture on its own cannot support a seamless recovery system for Mobile Routers. This is because there is no coherent control plane which encompasses today's diversified technologies and heterogeneous networks. The Ambient networking architecture with the concept of every networking entity as an 'Ambient Network' has introduced architectural principles that embrace heterogeneity in today's mobility environments [4].

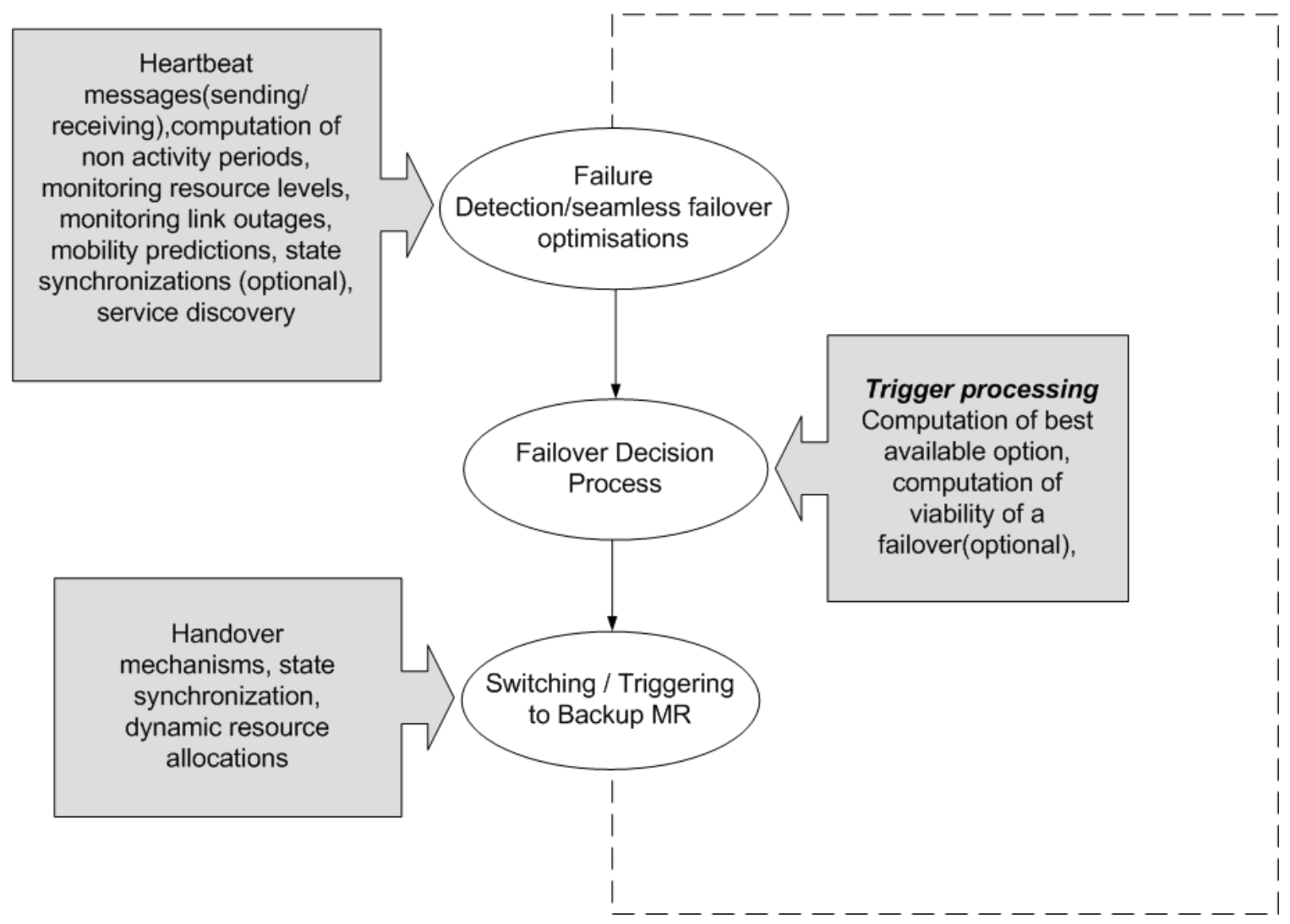

Fig. 5. Failover Functional Model

We derived this failover functional model after giving much consideration to the consequent requirements from the scenarios pertaining to the 3 different types of routing groups (managed, unmanaged and hybrid) with SMRs and USMRs. We have demonstrated the diversified nature of network mobility environments with Mobile Routers by considering 3 different scenarios with different types of routing functionalities and entities. In the following subsections we describe how various functionalities of the Ambient Control Space could jointly assist in the realization of failover management irrespective of which type of routing group or MRs are involved in the mobility environment. This emphasizes one of the key principles of the AN architecture which is handling heterogeneity in a seamless manner. 


\subsubsection{Information - oriented functionalities}

A common characteristic of all of the above mentioned functions is the need for a variety of information pertaining to each of the devices and to the networking environment as a whole. The liveliness of the devices, resources and capabilities of the devices and routing states for synchronization purposes are some of the information needed. Within ambient networking this information can be classified as context information i.e. information which represents the collection of any information that can be used to characterize the situation and/or operational state of an entity in different situations. The 'context provisioning' functionality of the ACS provides mechanisms to collect, store and disseminate context information. Since in the AN architecture any networking entity is also an AN, this information is available consistently regardless of the mobility environment. We do recognize that the granularity of information would change between different ANs, depending on the capability of the AN to produce this information (for example a mobile phone running on battery power (USMR) will not be able to contribute to the information base as much as a SMR). However, the important design principle in the AN architecture is that whatever information that is available can be used by other ANs in a similar manner. Therefore in failover management the USMRs, SMRs and any other networking entity in the mobility environment such as base stations, access points would be able to fulfill information oriented functionalities by making use of the "context provisioning" feature of the ACS. Moreover, QoS requirements pertaining to failovers which need to be dynamic and adaptive can be easily achieved by using the context provisioning functionality.

\subsubsection{Triggering functionalities}

The 'trigger processing' sub FA of 'mobility' FA acts upon inputs and decides whether the triggering input should be forwarded to other 'mobility' functions or ignored. Link outages, failure of an interface of the MR, draining of the battery etc. are triggering inputs that would need to be processed as part of a failover mechanism. Trigger processing would also have a conflict resolution process which could be very useful in the network mobility environment. For example triggers from two potential devices that could step in as backup routers would create a conflict and this would require mechanisms for conflict resolutions.

It is evident that these triggers (failures) come from different levels such as service levels, context levels and also from different layers. For example the absence of router advertisement messages in the network layer would be a trigger that indicates the failure of the active router. Since the trigger processing functionality would take into account any type of input, this simplifies failover management as there is no additional requirement to provide separate failover management mechanisms.

\subsubsection{Handover - oriented functionalities}

When a MR fails a handover to another device takes place, provided there exists another device capable of taking over the routing functionalities on behalf of the mobile network. Handovers that would occur in failover management are extremely diversified. Inter-interface handovers (between interfaces of a multimodal MR), inter-device handovers, inter-point of attachment handovers (between base stations, access points ) are types of handovers that we come across in network mobility. Seamless handovers is an essential requirement of all of the above handovers in the context of failover management of Mobile Routers. In order to provide seamless failovers it is necessary to give due consideration to handovers that are triggered by "urgency parameters". Functionalities for advanced handovers are considered within the 'Mobility' FA. Furthermore other functionalities within the ACS such as context provisioning, cross domain mobility etc. would encompass necessary functions in order to provide a seamless handover between different domains.

\subsubsection{Security - Oriented functionalities}

Security related functionalities are closely related to the aspects of trust relationship. Performing state synchronization, handover and other functions requires an existing trust between those entities. Existing trust relations can also be used and allow faster completion of failover functions. The security functional area within the ACS provides the necessary information whether a trust relationship is currently established or not. Accordingly, the failover function needs to establish a trust relation with the proxy/fallback MR before performing any other action. In the case of SMRs from different domains (in Scenario 1) the trust should be established in a proactive way to achieve apparently seamless operation. The security functionalities are also closely linked to the cross-domain functionalities introduced in 6.1.3. 
Table 1. Summary of requirements versus AN functional capabilities

\begin{tabular}{|l|l|l|}
\hline Requirement & Scenarios & Applicability of ACS functionalities \\
\hline $\begin{array}{l}\text { Location Predictions } \\
\text { Outage Predictions }\end{array}$ & Scenarios 1, 3 & Information oriented, Triggering oriented \\
\hline $\begin{array}{l}\text { Minimize the bandwidth overhead } \\
\text { for failover management protocols }\end{array}$ & Scenarios 1, 3 & $\begin{array}{l}\text { Information oriented, Triggering oriented, } \\
\text { Handover oriented }\end{array}$ \\
\hline $\begin{array}{l}\text { Find the minimum number of MRs } \\
\text { needed for maximum unperturbed } \\
\text { connectivity } \\
\text { Cross domain MR failover }\end{array}$ & Scenarios 1, 3 & $\begin{array}{l}\text { Information oriented, Triggering oriented, } \\
\text { Handover oriented, Security oriented }\end{array}$ \\
\hline $\begin{array}{l}\text { Minimize power consumption } \\
\text { overhead in failover management } \\
\text { protocols }\end{array}$ & Scenarios 2, 3 & Information oriented, Handover oriented \\
\hline $\begin{array}{l}\text { Service discovery } \\
\text { Known activation period (time until } \\
\text { operable) }\end{array}$ & Scenarios 2, 3 & $\begin{array}{l}\text { Security oriented, Information oriented, } \\
\text { Triggering oriented, Handover oriented }\end{array}$ \\
\hline $\begin{array}{l}\text { Dynamic state synchronization } \\
\text { Seamless state transition }\end{array}$ & Scenarios 2, 3 & $\begin{array}{l}\text { Information oriented, Triggering oriented, } \\
\text { Handover oriented }\end{array}$ \\
\hline Dynamically adaptive- QoS & Scenario 3 & $\begin{array}{l}\text { Information oriented, Triggering oriented, } \\
\text { Handover oriented, Security oriented }\end{array}$ \\
\hline
\end{tabular}

It can be viewed that the AN functionality areas cover well the requirements that elicited from the described AN mobility scenarios.

\section{Related Work}

It is evident that the related work on failovers for Mobile Routers is fragmented and many researchers are working on specific solutions, for example, how to reroute via another interface of a MR if the active interface fails. None of the research work on this area pertained to a much needed all encompassing architectural solution for this problem such as the one we envision with the AN project. Nevertheless we present here some research related to Mobile Routers that could be used in failover mechanisms such as multi-homing solutions.

The IETF NEMO working group [2] has introduced a network mobility architecture where the Mobile Router handles the mobility of the entire mobile network. This is achieved by the NEMO Basic Support protocol [5] which is a logical extension to the MIPv6 protocol [6]. The Mobile Router creates a bidirectional tunnel with its Home Agent and this tunnel is used for any communication to and from the mobile network. For this protocol to work without failures there needs to be 3 types of redundancies namely MR redundancy, Home Agent redundancy and Access Network redundancy. Multi-homing issues pertaining to IPv6 mobile networks are handled in [7], [8]. Paik et al in [9] describes a mechanism whereby a Home Agent is able to select the best MR.

The project Fleetnet [10] developed protocols for car-2-car based ad hoc networks. Accompanying to pure ad hoc communication the cars were able to communicate with the Internet using stationary roadside Internet Gateways. In the car a single router was deployed handling all the communication with other cars and Internet Gateways. The field of failover management was only investigated with respect to the dynamicity of a managed routing group. The scenario introduced with the hybrid routing group described in section 3.3 was not covered in that project.

The BRAIN [11] and MIND [12] projects developed protocols for extending edge networks by using devices provided by ad hoc fringes as MIND Access Network Wireless Routers (ANWR). In combination with the BRAIN Access Routers (BAR) and BRAIN Mobility Gateways, a mobility infrastructure which supports highly mobile devices was designed; however, the existence of several MRs was not supported in these projects. 


\section{Conclusions}

It is evident that the IP architecture is not proficient enough to provide seamless failovers for Mobile Routers in different network mobility environments. As demonstrated by the variety of mobile network types and corresponding analysis of the failover scenarios, it is necessary to introduce an all encompassing simple networking architecture in order to facilitate mechanisms such as failovers. We have demonstrated that the Ambient Networking architecture which introduces a thick but simple control plane would be able to handle seamless failovers for Mobile Routers.

It is necessary to note that within this paper our intention was not to specify a certain solution, but rather to show how the ambient networking architecture would pave the way for failover management of Mobile Routers in a seamless manner.

\section{Acknowledgements}

This document is a byproduct of the Ambient Networks Project, partially funded by the European Commission under its Sixth Framework Programme. It is provided "as is" and without any express or implied warranties, including, without limitation, the implied warranties of fitness for a particular purpose. The views and conclusions contained herein are those of the authors and should not be interpreted as necessarily representing the official policies or endorsements, either expressed or implied, of the Ambient Networks Project or the European Commission. The authors would like to thank Eisl Jochen and Vijay Sivaraman for reviewing this paper on numerous occasions.

\section{References}

1. http://www.ambient-networks.org/

2. Perkins C., "IP Mobility support for IPv4,” RFC 3344, IETF, August 2002.

3. http://www.ietf.org/html.charters/nemo-charter.html

4. N. Niebert, A. Schieder, H. Abramowicz, G. Malmgren, J. Sachs, U. Horn, Ch. Prehofer and H. Karl, „Ambient Networks: An Architecture for Communication Networks beyond 3G“, IEEE Wireless Communications, April 2004.

5. Devarapalli V., Wakikawa R., Petrescu A., Thubert P., "NEMO Basic Support Protocol” (draft-ietf-nemo-basicsupport-03.txt), Internet Draft, IETF, June 2004, Work in Progress.

6. Johnson D., Perkins C., Arkko J., “Mobility Support in IPv6”, RFC 3775, IETF, June 2004.

7. Kuntz R., Paik E., Tsukada M., Ernst T., Mitsuya K., "Evaluating Multiple Mobile Routers and Multiple NEMOPrefixes in NEMO Basic Support” (draft-kuntz-nemo-multihoming-test-00.txt), Internet Draft, IETF, July 2004, Work in Progress.

8. Ng C., Paik E., Ernst T., “Analysis of Multihoming in Network Mobility Support” (draft-ietf-nemo-multihomingissues-00.txt), Internet Draft, IETF, July 2004, Work in Progress.

9. Paik E., Cho H., Ernst t., Choi Y., "Load Sharing and Session Preservation with Multiple Mobile Routers for Large Scale Network Mobility," 18th International Conference on Advanced Information Networking and Applications (AINA 2004), IEEE Computer Society Press, Fukuoka, Japan, March 2004, pp. 393-398.

10. http://www.et2.tu-harburg.de/fleetnet/

11. IST 1999-10054 Project BRAIN, Deliverable D2.2, March 2001

12. IST 2000-28584 Project MIND, Deliverable D2.2, November 2002 